\title{
Analysis of 5 years of morbidity and mortality conferences in a metropolitan South African trauma service
}

\author{
V Y Kong, MB ChB, MSc, PhD, MRCS (Eng); D L Clarke, MMedSci, MBA, MPhil, PhD, FCS (SA) \\ Pietermaritzburg Metropolitan Trauma Service, Department of Surgery, University of KwaZulu-Natal, Pietermaritzburg, South Africa
}

Corresponding author: D L Clarke (damianclar@gmail.com)

\begin{abstract}
Background. Since 2008 the Pietermaritzburg Metropolitan Trauma Service (PMTS) has run a structured, self-reporting, metropolitan morbidity and mortality conference (MMC). In 2012 a hybrid electronic medical registry (HEMR) was introduced to capture routine data and to generate reports on morbidity and mortality. This paper reviews our experience in setting up a metropolitan MMC and compares the quality of the reported morbidity data from the pre- and post-HEMR era.

Methods. We compared data from the MMC before and after the introduction of the HEMR to audit the impact of these meetings on the reporting and analysis of surgical morbidity and mortality in our service.

Results. During the 4-year period from 2008 to 2011, a total of 208 MMCs were held. A total of 10682 patients were admitted by the PMTS during that period, of whom $87 \%$ were males, and the mean age was 26 years. Penetrating trauma accounted for $40.9 \%(4344 / 10628)$ of the total workload. A total of 432 (4.1\%) morbidities were identified. Of these, 36.6\% (158) were related to human error, 32\% (138/432) were related to surgical pathologies and the remaining $31.9 \%$ (136/432) were related to systemic diseases. There was an exponential increase in the reporting of morbidity each year. The total in-hospital mortality was 3\% (358/10 682). Following the introduction of the HEMR, from 2012 to 2014, 6217 patients were admitted. A total of 1314 (21.1\%) adverse events and 315 (5.1\%) deaths were recorded by the HEMR. The adverse events were divided into 875 'pathology-related' morbidities and 439 'error-related' morbidities.

Conclusions. The development of the MMC led to increased reporting of morbidity and mortality. The introduction of the HEMR resulted in a dramatic improvement in the capturing of morbidity and mortality data, suggesting that a paper-based self-reporting system tends to underestimate morbidity. Over one-third of all morbidities were related to human error. Common morbidities have been identified.

S Afr Med J 2016;106(7):695-698. DOI:10.7196/SAMJ.2016.v106i7.10549
\end{abstract}

Over the last two decades there has been an increased awareness of the significant contribution of human error to mortality and morbidity in trauma and acute care surgery..$^{[1-5]}$ The culture of surgical practice is, however, often inimical to the open and transparent reporting of surgical adverse events, error and morbidity. This creates difficulties in developing strategies to address human error and to reduce surgical and trauma-related morbidity. The traditional forum for identifying and discussing surgical complications is the morbidity and mortality conference (MMC) and many authors feel that the MMC should be used to drive quality improvement programmes in surgical services. ${ }^{[6-8]}$ The Pietermaritzburg Metropolitan Trauma Service (PMTS) established a weekly metropolitan morbidity and mortality meeting in 2007. This has run since then and has been a predominantly paperbased system which relied on self-reporting. ${ }^{[3-5]}$ However, selfreporting of morbidity is generally believed to result in a significant underestimation of morbidity. Subsequent to the institution of the MMC in 2007 we introduced a hybrid electronic medical record system (HEMR) with a dedicated interface for capturing morbidity in 2012. We published our initial experience with the HEMR morbidity interface in $2014 .^{[9,10]}$ The aim of this paper is to review our earlier experience in setting up a metropolitan MMC and to compare it with our more recent experience. Data captured by this conference between 2007 and 2011 were reviewed to categorise morbidity and error associated with trauma care in our environment. These data were compared with recently published data from the HEMR dating from 2012 to 2014 to see how the introduction of an electronic interface to capture data impacted on morbidity data ${ }^{[9,10]}$ A secondary aim of the project is to identify common errors and morbidities in trauma care. It is hoped that this information will allow us to develop targeted educational and quality-improvement programmes to reduce error and morbidity.

\section{Methods \\ Setting}

Pietermaritzburg is the largest city in the interior of KwaZuluNatal (KZN) Province. The PMTS was established in 2006 with the intention of providing comprehensive trauma care to the city of Pietermaritzburg and the entire western part of KZN province. The PMTS serves a catchment population of over three million people.

\section{The metropolitan MMC}

The combined metropolitan MMC for trauma was established in 2008. This is held on a weekly basis and every trauma patient admitted to the PMTS is discussed. Emphasis is placed on any morbidity or mortality encountered and each operative case is discussed in detail. A PowerPoint presentation is generated for each conference. The conference is attended by all staff and is chaired by the trauma specialists within the PMTS. A peer review process by the senior staff is used to classify all complications and errors. All trauma cases are classified according to the type of trauma and the region injured. Morbidity is classified as disease related, iatrogenic or error related. A specific note is made of the complications associated with two common index procedures, intercostal drain (ICD) insertion and central venous line $(\mathrm{CVL})$ placement.

A paper-based trauma registry was instituted in 2006, and ethical approval to maintain this registry was provided by the Biomedical Research Ethics Committee (BREC) of the University of KwaZuluNatal (BE 207/09). In addition to the paper-based trauma registry, each PowerPoint presentation is stored. Data are extracted from 
each MMC presentation into a Microsoft Excel spreadsheet which forms part of the departmental record. In 2012, a new format to the conference was introduced, a new electronic surgical registry (ESR) and ultimately an HEMR. This also has ethics approval (BE 221/13). This article focuses on the initial 5 years of our MMC from 2008 to 2011, prior to the introduction of the ESR and the HEMR, and compares the reported morbidity from these two periods.

\section{Data review}

Data that were reviewed included basic demographics and the total number of admissions, as well as the mechanism of injury. The mechanism of injury was separated into blunt trauma (BT), penetrating trauma (PT), and other trauma (OT). Trauma was sub-classified according to the anatomical region injured. All operative procedures were recorded and each operation was classified as either an emergency or a non-emergency operation.

\section{Results \\ Demographics}

During the 5-year study period, a total of 208 MMCs were held. A total of 10628 patients were admitted to the PMTS during the same period, of whom $9300(87.5 \%)$ were males. The mean age was 26 years. A total of $422(4.0 \%)$ of all patients required admission to the intensive care unit.

\section{Mechanism of injury}

Of the 10682 patients admitted, 6288 (58.9\%) sustained BT, 4344 (40.1\%) sustained PT, with the remaining 50 patients' trauma classified as miscellaneous injuries (OT). Table 1 shows the different mode of injury in the 6288 patients with BT. Table 2 shows the injuries by anatomical region. Of the 4344 patients with PT, 3736 (86.0\%) had stab injuries and the remaining $608(14.0 \%)$ gunshot injuries.

\section{Operations}

A total of 1452 operations were performed: 910 (62.7\%) were emergency operations, and the remaining $542(37.3 \%)$ were non-

\section{Table 1. BT: mode of injury $(N=6288)$}

\begin{tabular}{ll}
\hline Mode & $\boldsymbol{n}(\%)$ \\
\hline Assault & $3748(59.6)$ \\
RTA & $1828(29.1)$ \\
Crush injury & $362(5.8)$ \\
Fall & $248(3.9)$ \\
RTA = road traffic accident. &
\end{tabular}

emergency operations. Table 3 summarises the different operations.

\section{Morbidity and mortality}

A total of 432 morbidities were identified. The total in-hospital mortality rate was $3.4 \%$
(358/10 682). Iatrogenic injury accounted for 274 (63.4\%) morbidities, and error was involved in 158 (37.6\%). There was an exponential increase in the reporting of morbidity over the period under review, and Fig. 1 shows this temporal trend from 2008 to 2011.

Table 2. Anatomical distribution of trauma ( $N=10632)$

\begin{tabular}{llll}
\hline Region & BT (N=6 288), $\boldsymbol{n}(\%)$ & SWs $(\boldsymbol{N = 3 ~ 7 3 6 ) , ~} \boldsymbol{n}(\%)$ & GSWs $(\boldsymbol{N = 6 0 8 ) , \boldsymbol { n } ( \% )}$ \\
\hline Multi-regional & $1342(21.3)$ & $308(8.2)$ & $26(4.3)$ \\
Head & $3552(56.5)$ & $226(6.0)$ & $30(4.9)$ \\
Face & $372(5.9)$ & $74(2.0)$ & $18(3.0)$ \\
Neck & $96(1.5)$ & $382(10.2)$ & $28(4.65)$ \\
Chest & $386(6.1)$ & $1740(46.6)$ & $200(32.9)$ \\
Abdomen & $344(5.5)$ & $812(21.7)$ & $202(33.2)$ \\
Limbs & $196(3.1)$ & $194(5.2)$ & $104(17.1)$ \\
SWs = stab wounds; GSWs = gunshot wounds. & &
\end{tabular}

Table 3. Operations: overview and indications $(N=1452)$

\begin{tabular}{llllll}
\hline Operation & $\boldsymbol{n}(\%)$ & BT $(\boldsymbol{n}=\mathbf{1 7 8})$ & SWs $(\boldsymbol{n}=\mathbf{7 1 6})$ & GSWs $(\boldsymbol{n}=\mathbf{2 7 2})$ & Others $(\boldsymbol{n}=\mathbf{6})$ \\
\hline Laparotomy & $602(41.5)$ & 142 & 306 & 154 & 0 \\
$\begin{array}{l}\text { Relook } \\
\text { laparotomy }\end{array}$ & $138(9.5)$ & 26 & 68 & 40 & 2 \\
Laparoscopy & $94(6.5)$ & 4 & 86 & 4 & 0 \\
$\begin{array}{l}\text { Thoracotomy } \\
\text { Video-assisted }\end{array}$ & $742(8.4)$ & 0 & 102 & 20 & 0 \\
thoracoscopy & & 4 & 56 & 12 & 2 \\
$\begin{array}{l}\text { Neck } \\
\text { exploration }\end{array}$ & $64(4.4)$ & 0 & 54 & 10 & 0 \\
$\begin{array}{l}\text { Vascular } \\
\text { exploration }\end{array}$ & $80(5.5)$ & 2 & 44 & 32 & 2 \\
$\begin{array}{l}\text { Tracheostomy } \\
\text { Miscellaneous }\end{array}$ & $70(4.8)$ & - & - & - & - \\
& $208(14.3)$ & - & - & - & -
\end{tabular}

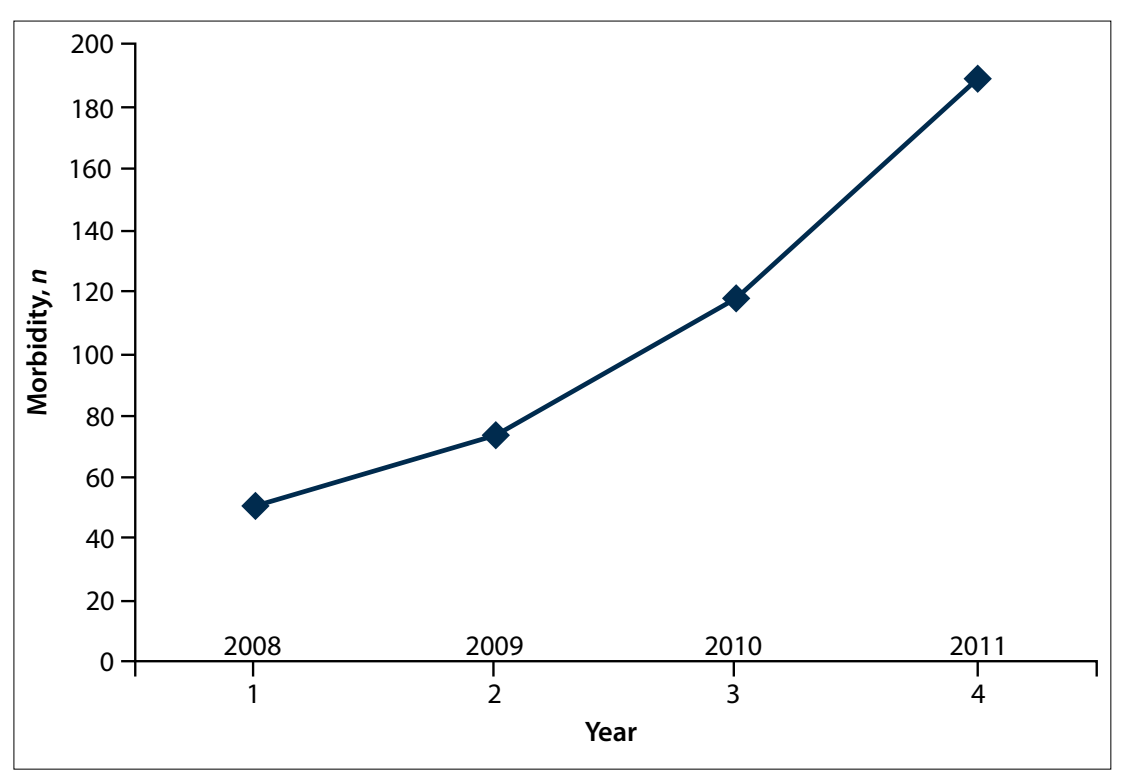

Fig. 1. Morbidity reported by year (2008 - 2011). 


\section{Morbidity related to thoracic trauma}

A total of 29 thoracic-trauma-related morbidities were discussed during the 5-year period. In 25 patients there was delayed clinical recognition of a tension pneumothorax (TPTX). Of this cohort 12 were discovered on chest X-ray (CXR), 8 on computed tomography (CT) scan without an initial CXR, and 5 in the operating room. Of the 12 patients who had a TPTX discovered on CXR, 7 (58.3\%) died. Three of the 7 patients who died were referred from another hospital with a CXR film accompanying them showing a TPTX, and all were dead on arrival. Three other patients who presented directly to our unit were sent to the radiology suite for a CXR following initial assessment, and died shortly afterwards. All these three patients had CXRs taken prior to their death that showed TPTXs. A patient died in the operating room following a sternotomy and repair of a penetrating cardiac injury. He arrested as a result of an unrecognised TPTX.

Four patients sustained iatrogenic visceral injuries as a direct result of ICD insertion. Three of these patients sustained a diaphragmatic perforation, which required operative repair. The fourth patient sustained an acute diaphragmatic rupture with intrathoracic gastric herniation following a road traffic accident. This was not recognised on the initial CXR and he sustained a gastric perforation following ICD insertion. He subsequently underwent a laparotomy. Two patients sustained iatrogenic visceral injuries to the intercostal vessels during insertion of an ICD.

\section{Morbidity related to abdominal trauma}

There were 82 documented morbidities related to the management of abdominal trauma. Among the $6288 \mathrm{BT}$ patients, error of assessment was reported in 75 patients (1\%), resulting in therapeutic delays of over 12 hours. In the delayed group there were 4 bladder injuries, 24 small-bowel perforations, 2 duodenal injury, 2 infarctions of the ascending colon and 8 small-bowel mesenteric avulsions. A total of 7 injuries were not detected during the 602 index laparotomies (1\%); these included 2 lacerations of the gallbladder, 2 duodenal injuries, 2 small-bowel injuries and a missed vena-caval injury. The operating surgeon elected not to explore a central haematoma, and this injury was ultimately fatal.

\section{Morbidity related to ICD placement}

A total of 1050 ICDs were inserted over this 5-year period and a total of 203 (19\%) ICD-related complications were documented. These included kinking of the ICD, subcutaneous insertion, incomplete insertion and inadequate fixation resulting in dislodgement. Four patients $(2 \%)$ sustained iatrogenic visceral injuries as a direct result of ICD insertion.

\section{Morbidity related to central line insertion}

A total of 1020 CVLs were placed and there were 89 CVL-related morbidities during the period under review. A total of $45(4 \%)$ post-CVL insertion pneumothoraces were reported. A single central line was placed into the pleural cavity and the patient received total parental nutrition (TPN) into the pleural space. In 9 patients $(<1 \%)$ arterial puncture lead to a haematoma. Twenty (2\%) CVLs became dislodged and in $19(<2 \%)$ the CVL was not placed into the superior vena cava (SVC) as intended.

\section{General morbidity}

During the 5-year period 29 general morbidities were reported. Sepsis accounted for $3.0 \%$ (13/432) general morbidities, and was equally divided between surgical site sepsis and pneumonia. Failure to review investigations was a problem in 5 patients and included failure to review a CXR, which showed a misplaced CVL (1), a pneumothorax post-CVL insertion (1), a collapsed lung due to a mucus plug (1) misplaced feeding tube (1) and a retained blade (1). A patient with a retained weapon in his leg was discharged home and returned later with a suppurating wound. Inappropriate drug prescription was a problem in 11 patients and included the administration of nephrotoxic agents to patients with acute kidney injury in 4 patients, the omission of thromboprophylactic agents or antibiotics in 4 and incorrect dosing in 3 .

\section{Morbidity captured by the HEMR}

From 2012 to 2014, a total of 6217 patients were admitted to our surgical service. A total of 1314 (21.1\%) adverse events and 315 (5.1\%) deaths were recorded. The adverse events were divided into 875 'pathology-related' morbidities and 439 'error-related' morbidities. Pathology-related morbidities included 725 systemic complications and 150 operative complications. Error-related morbidities included 158 iatrogenic injuries and 24 missed injuries. Error accounted for 439 (33.4\%) of the total number of adverse events. Table 4 compares the data from the pre-HEMR meetings and the post-HEMR meetings.

\section{Discussion}

Surgical morbidity is generally under-reported and there are many reasons why this is the case. ${ }^{[11,12]}$ These include a culture of blame, which makes staff reluctant to report morbidity, as well as inadequate methods of collecting data on morbidity and of classifying and categorising such data. ${ }^{[13-15]}$ James Reason, a pioneer of the study of human error, has identified four critical elements of an effective organisational safety culture, namely a culture that is committed to reporting morbidity and which is, just, flexible, and open to learning. ${ }^{[1,2]}$ The challenge remains for healthcare systems to develop such a culture, and many commentators feel that the surgical morbidity and mortality meeting is ideally placed to lead this process. ${ }^{[-8]}$ Changing the culture of an organisation is a difficult task which requires a longterm commitment to ongoing educational initiatives to explain the principles of error theory and to stress the fact that failure must be understood as systemic rather than due to individual behaviour. Our ongoing efforts have been effective, as evidenced by the exponential increase in reporting of morbidity over the initial 5-year period of the metropolitan M\&M meeting. Fig. 1 demonstrates this increased rate of reporting of morbidity over the 5-year period under review.

Most authors feel that self-reporting of morbidity alone is ineffective and a systematic and comprehensive approach to collating morbidity data is needed. ${ }^{[11,12]}$ This is supported by the fact that the implementation of the HEMR resulted in reported morbidity increasing from $4 \%$ to over $20 \%$. Table 4 compares the situation before and after the introduction of the HEMR. The dramatic increase in reported morbidity was due to the increased reporting of sepsis and especially hospital-acquired pneumonia. Taxonomies to classify and

\begin{tabular}{l}
$\begin{array}{l}\text { Table 4. A comparison of the reported morbidity and } \\
\text { mortality pre- and post-introduction of the HEMR }\end{array}$ \\
\hline \\
\begin{tabular}{lll} 
Pre-HEMR & Post-HEMR \\
& 2008 - 2011 & 2012 - 2014 \\
\hline Patients, $N$ & 10682 & 6217 \\
Morbidity, $n(\%)$ & $432(4.1)$ & $1314(21.1)$ \\
Mortality, $n(\%)$ & $358(3.4)$ & $315(5.1)$ \\
Error, $n / N(\%)$ & $158 / 432(36.6)$ & $439 / 1314(33.4)$ \\
Iatrogenic, $n / N(\%)$ & $292 / 432(67.6)$ & $158 / 1314(12.0)$ \\
Missed injury, $n / N(\%)$ & $7 / 602(1.2)$ & $24 / 1314(1.8)$ \\
Sepsis, $n / N(\%)$ & $13 / 432(3.0)$ & $301 / 1314(22.9)$
\end{tabular}
\end{tabular}


categorise morbidity are frequently inadequate and, when available, poorly understood, and this further complicates the reporting and capturing of accurate data on morbidity. ${ }^{[13-15]}$ A good example of this is the lack of consensus on the most appropriate way to classify surgical complications such as surgical site sepsis and anastomotic failure. ${ }^{[13]}$ The major increase in reporting with the HEMR has been around systemic complications such as sepsis and pneumonia. In the pre-HEMR system sepsis was reported in only 13 patients. With the HEMR system this had increased to 301 patients with sepsis; among these nosocomial pneumonia and surgical site infection accounted for 100 and 96 cases, respectively. This suggests that these morbidities in particular are grossly under-represented in paper-based self-reporting systems. There was no increase in the reported incidence of mortality over the two time periods. This reflects the fact that a surgical fatality is a definitive and dramatic endpoint, which tends to get reported, whereas morbidity is far more difficult to capture and quantify. ${ }^{[13-15]}$

The important contribution of human error to surgical adverse events is increasingly recognised and our data suggest that over a third (158/432) of all morbidities were related to human error rather than surgical or systemic disease. This has remained constant with the introduction of the HEMR, which reported human error as a cause of $33 \%$ of all adverse events. ${ }^{[1-4]}$ These findings highlight the need for both general error awareness training and specific interventions for a number of common clinical scenarios and procedures. ${ }^{[16,17]}$ In addition modern taxonomies of error and modern classifications of morbidity must be used to help staff understand where in the process of care error intervened. ${ }^{[1,2,4,5,8,13,15]}$

The most common morbidity associated with thoracic trauma is the failure to recognise and treat a tension pneumothorax and the failure to diagnose acute diaphragmatic herniation. Thoracic trauma is a common surgical condition in our institutions and there are a number of courses designed to equip junior staff to manage these injuries. The most wellknown course is the Advanced Trauma Life Support (ATLS) course of the American College of Surgeons. Despite the wide penetrance of ATLS in our environment, we still see a significant morbidity associated with thoracic trauma. ${ }^{[17]}$ This is secondary to both diagnostic failure and procedural failure, and evidenced by the observation that iatrogenic morbidity secondary to CVL placement and ICD insertion are common problems. These failures can be considered as protocol violations, as failure to follow ATLS guidelines resulted in morbidity and even mortality. It is apparent that educational courses in isolation will not reduce the incidence of surgical morbidity and they need to be included as part of a multifaceted quality improvement programme. ${ }^{[3]}$ This is analogous to the fact that good traffic regulations by themselves will not reduce traffic-related accidents and a combination of enforcement and mechanical interventions such as traffic calming speed humps are required to improve traffic safety. The development of preoperative 'tickbox' style checklists has been shown to help reduce operative mortality and may have a major role to play in reducing morbidity associated with these common procedures. ${ }^{[18,19]}$

The morbidity most commonly associated with blunt abdominal trauma is delayed recognition of the need for laparotomy. Hollow visceral injury following blunt abdominal trauma has an indolent presentation, as mesenteric avulsion may initially be asymptomatic as the avulsed segment only becomes ischaemic after several hours. Failure to review special investigations is a problem, as evidenced by the missed retained blade and the missed pneumothoraces post CVL insertion. The incidence of missed injury at operation has remained constant at just under 1.5\%, in all reports from our institution from 2008 until the present. ${ }^{[3,4]}$ These morbidities are much less common than those associated with thoracic trauma, ICD insertion and CVL placement. The most appropriate educational interventions for these errors and morbidities will most likely be general error awareness training to encourage staff to be error aware. These programmes must encourage staff to constantly recycle information while actively excluding likely complications and clinical scenarios.

\section{Conclusion}

The institutionalisation of a structured MMC resulted in increased reporting of morbidity in our trauma service. However, the reliance on self-reporting and paper-based systems still resulted in gross under-reporting of morbidity, as shown when data are compared with the post-HEMR era. The patterns of morbidity have largely remained constant between the two eras although pneumonia and surgical site infection seem to have been particularly poorly captured under the previous system. We have identified common clinical scenarios and procedures that are associated with error and morbidity. It is hoped that analysis of the cases discussed at the MMC can inform educational initiatives that directly target human error.

\section{References}

1. Reason J. Human error: Models and management. BMJ 2000;18:320(7237):768-770. DOI:10.1136/ bmj. 320.7237 .768

Reason J. Understanding adverse events: Human factors. Qual Health Care 1995;4(2):80-8

3. Clarke DL. Trauma quality improvement: The Pietermaritzburg Metropolitan Trauma Service experience with the development of a comprehensive structure to facilitate quality improvement in rural trauma and acute care in KwaZulu-Natal, South Africa. S Afr Med J 2015;105(2):92-95. DOI:10.7196/samj. 8792

4. Clarke DL, Gouveia J, Thomson SR, Muckart DJ. Applying modern error theory to the problem of missed injuries in trauma. World J Surg 2008;32(6):1176-1182. DOI:10.1007/s00268-008-9543-7

Clarke DL, Aldous C, Thomson SR. The implications of the patterns of error associated with acute trauma care in rural hospitals in South Africa for quality improvement programs and trauma trauma care in rural hospitals in South Africa for quality

6. Davies J, Chintapatla S, Miller G. Developing a weekly patient safety and quality meeting in a mediumsized GI surgical unit in the United Kingdom. Saf Surg 2014;8(1):6. DOI:10.1186/1754-9493-8-6

7. Higginson J, Walters R, Fulop N. Mortality and morbidity meetings: An untapped resource for . Higginson J, Walters R, Fulop N. Mortality and morbidity meetings: An untapped resource for
improving the governance of patient safety? BMJ Qual Saf 2012;21(7):576-585. DOI:10.1136/ improving the gover

8. Clarke DL, Furlong H, Laing GL, et al. Using a structured morbidity and mortality meeting to understand the contribution of human error to adverse surgical events in a South African regional hospital. S Afr J Surg 2013;51(4):122-126. DOI:10.7196/sajs.1537

9. Laing GL, Bruce JL, Skinner DL, et al. Development, implementation and evaluation of a hybrid electronic medical record system specifically designed for a developing world surgical service. World J Surg 2014;38(6):1388-1397. DOI:10.1007/s00268-013-2438-2

10. Laing G, Bruce J, Skinner D, et al. Using a hybrid electronic medical record system for the surveillance of adverse surgical events and human error in a developing world surgical service. World J Surg 2015;39(1):70-79. DOI:10.1007/s00268-014-2766-x

11. Etchegaray JM, Gallagher TH, Bell SK, Dunlap B, Thomas EJ. Error disclosure: A new domain for safety culture assessment. BMJ Qual Saf 2012;21 (7):594-599. DOI:10.1136/bmjgs-2011-000530

2. Kronman AC, Paasche-Orlow M, Orlander JD. Factors associated with disclosure of medical errors by Kronman AC, Paasche-Orlow M, Orlander JD. Factors associated with disclosure
house staff. BMI Qual Saf 2012;21(4):271-278. DOI:10.1136/bmjgs-2011-000084 13. Chang A, Schyve PM, Croteau RJ, et al. The JCAHO patient safety event taxonomy: A standardized
terminology and classification schema for near misses and adverse events. Int J Qual Health Care terminology and classification schema for near

14. Bruce J, Russell EM, Mollison J, Krukowski ZH. The measurement and monitoring of surgical adverse events. Health Technol Assess 2001;5(22):1-194.

15. Dindo D, Demartines N, Clavien PA. Classification of surgical complications: A new proposal with evaluation in a cohort of 6336 patients and results of a survey. Ann Surg 2004;240(2):205-213. DOI:10.1097/01.sla.0000133083.54934.ae

16. Aldous CM, Searle R, Clarke DL. An educational programme for error awareness in acute trauma for junior doctors. Afr J Health Professions Educ 2014;6(2):161-164. DOI:10.7196/AJHPE.350

17. Kong VY, Oosthuizen GV, Sartorius B, Keene CM, Clarke DL. Correlation between ATLS training and junior doctors' anatomical knowledge of intercostal chest drain insertion. Surg Educ 2015:72(4):600605. DOI:10.1016/j.jsurg.2015.01.022

18. Weiser TG, Haynes AB, Dziekan G, Berry WR, Lipsitz SR, Gawande AA. Safe Surgery Saves Lives Investigators and Study Group. Effect of a 19-item surgical safety checklist during urgent operations investigators and Study Group. Effect of a 19-item surgical safety checklist during urgent operations 9. Civil I. Tube thoracostomy in trauma: Is it time for a checklist for test tubes? Injury 2013;44(9):11431144. DOI:10.1016/j.injury.2013.06.001 